\title{
Short-term instabilities in $\gamma$ Velorum: a search for strange modes in variable stars
}

\author{
John Bentley, P.L. Cottrell \\ Mt. John University Observatory, Department of Physics \& Astronomy, \\ University of Canterbury, Private Bag 4800, Christchurch, New Zealand
}

\begin{abstract}
Strange mode instabilities in Wolf-Rayet stars have been proposed as the cause of clumping in the stellar wind. This, in turn, is suggested as a cause of line profile variations found on short time scales. High-frequency, short-duration, time-series spectroscopy was performed on the Wolf-Rayet star $\gamma$ Velorum and analysis confirmed the results of a previous study of $\gamma$ Velorum's stellar wind which showed that the wind is indeed clumped, most likely from the predicted strange mode pulsations.
\end{abstract}

\section{Introduction}

Investigations of strange pulsational modes show that they are common in very high mass stellar models. They have also been found to occur in some lower mass stars and the investigations predict short-term line profile variability as a result. Wolf-Rayet models have been found to be the most strongly affected by these pulsational modes (Kiriakidis et al. 1996). These pulsations would force the normally homogeneous stellar wind into clumps. The presence of clumped winds is thought to be shown by line profile variations, specifically variable sub-peaks on emissions lines. The line profile variations of emission lines can be used to probe the structure and behaviour of a radiation driven, clumped stellar wind. A regiment of high-frequency spectroscopic sampling of the WolfRayet star $\gamma$ Velorum was undertaken. These data were analysed to look for line profile variations similar in behaviour to what is predicted for clumped, radiation-pressure driven winds (Lépine, Eversberg \& Moffat 1999).

\section{Line profile variations}

Over short time scales, homogeneous stellar winds have a generally stable emission line profile, unlike the emission lines of clumped winds which are constantly changing in structure. These changes can be up to a few percent of the intensity of the emission line. Glatzel (2001) has suggested that unstable strange modes could develop at the core-envelope boundary causing sound waves to be emitted that would compress the stellar wind into clumps. The amount of energy contributing to the emission line profile would then depend on the number and direction of clumps passing through the line-emission region of the star. As the clumps are driven further away by the intense radiation pressure of the star, they would cool and emit less radiation. The behaviour of the line profile 


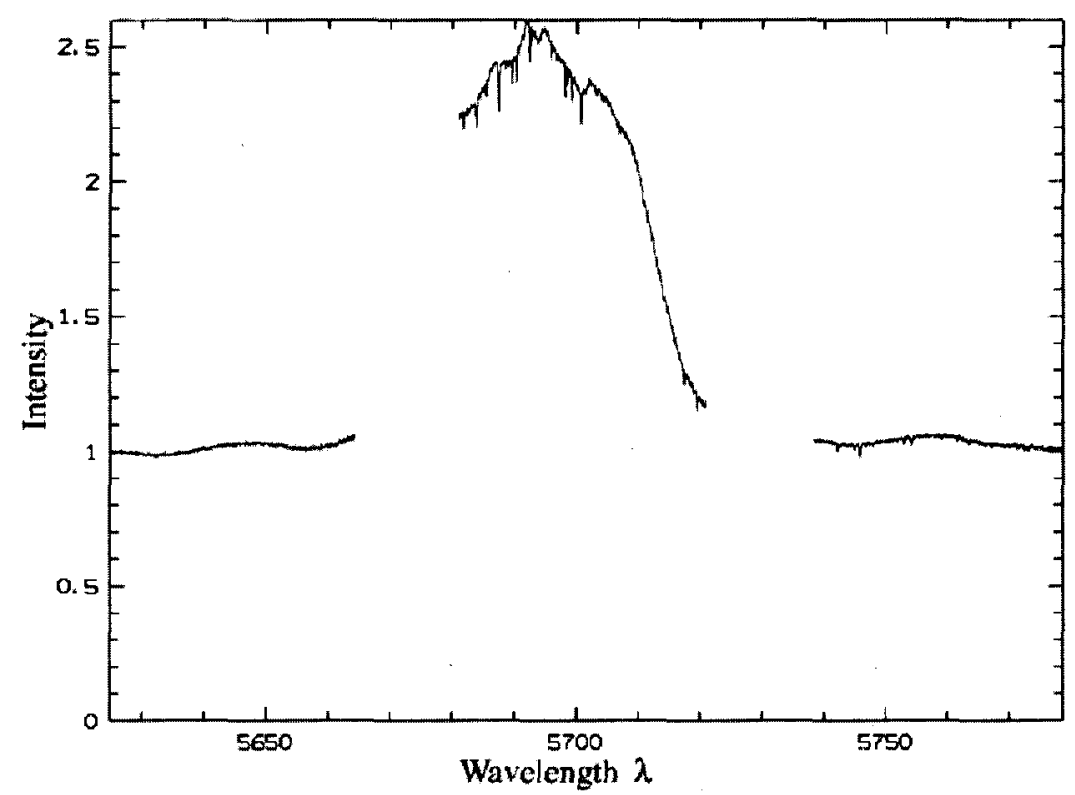

Figure 1. Normalised spectrum of the C III $\lambda 5696 \AA$ emission line in $\gamma$ Velorum.

variations is predicted to be small sub-peaks forming at the centre of emission lines that would decay in intensity as they moved away from the line centre. This behaviour in the spectra of Wolf-Rayet stars would show the presence of a clumped wind and the effect of strange modes.

\section{Observations and data reduction}

Spectroscopic observations of $\gamma$ Velorum were obtained at the Mount John University Observatory (MJUO) using the 1-m telescope and the HERCULES spectrograph (Hearnshaw et al. 2002). A typical night's dataset consisted of 30-60-s exposures obtained continuously over a $3-5 \mathrm{hr}$ period. Thorium-Argon wavelength calibration images were obtained every 5-10 min. Emission excesses were calculated using the instantaneous line variations gradient method from Lépine et al. (1999). Several emission lines were studied and the C III $\lambda 5696 \AA$ line was chosen for more detailed analysis (Fig. 1).

\section{Results}

Analysis of the MJUO 2003 data shows the expected behaviour in the line profile variations. It also agrees with similar studies of $\gamma$ Velorum's C III $\lambda 5696 \AA$ emission line by Lepine et al. (1999). The grey scale residual spectra of MJUO data (Fig. 2a) shows emission excess forming near the centre of the emission line and moving toward higher Doppler velocities $(\xi)$. A comparison with the 

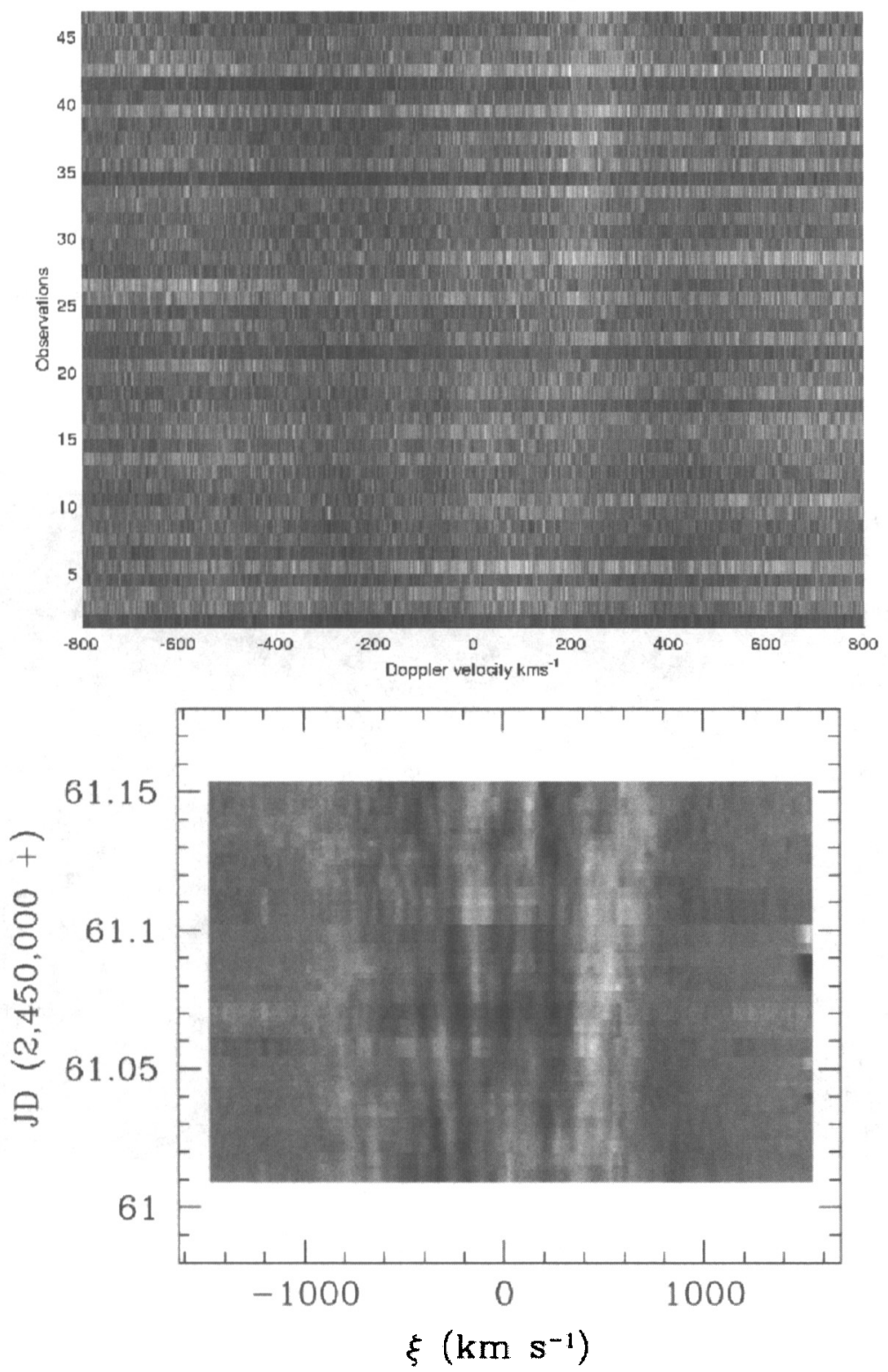

Figure 2. Contour plots showing line profile variations in the CIII $\lambda 5696 \AA$ emission line in $\gamma$ Velorum: (a: top) MJUO 2003 data (b: bottom) Lépine et al. (1999) data. 
Lépine et al. (1999) observations, is shown in Fig. 2b. Since the wind is being accelerated, the higher Doppler velocities correspond to larger radial distances from the star. At larger distances, the clumps would cool and therefore emit less. This is consistent with the decay in intensity shown in Fig. 2.

\section{References}

Glatzel, W. 2001, Rev. Mod. Astr., 14, 245

Hearnshaw, J.B., Barnes, S.I., Kershaw, G.M., Frost, N., Graham, G., Ritchie R., Nankivell G.R. 2002, Experimental Astronomy, 13, 59

Kiriakidis, M., Glatzel, W., Fricke, K.J. 1996, MNRAS, 281, 406

Lépine, S., Eversberg, T., Moffat, A.F.J. 1999, AJ, 117, 1441

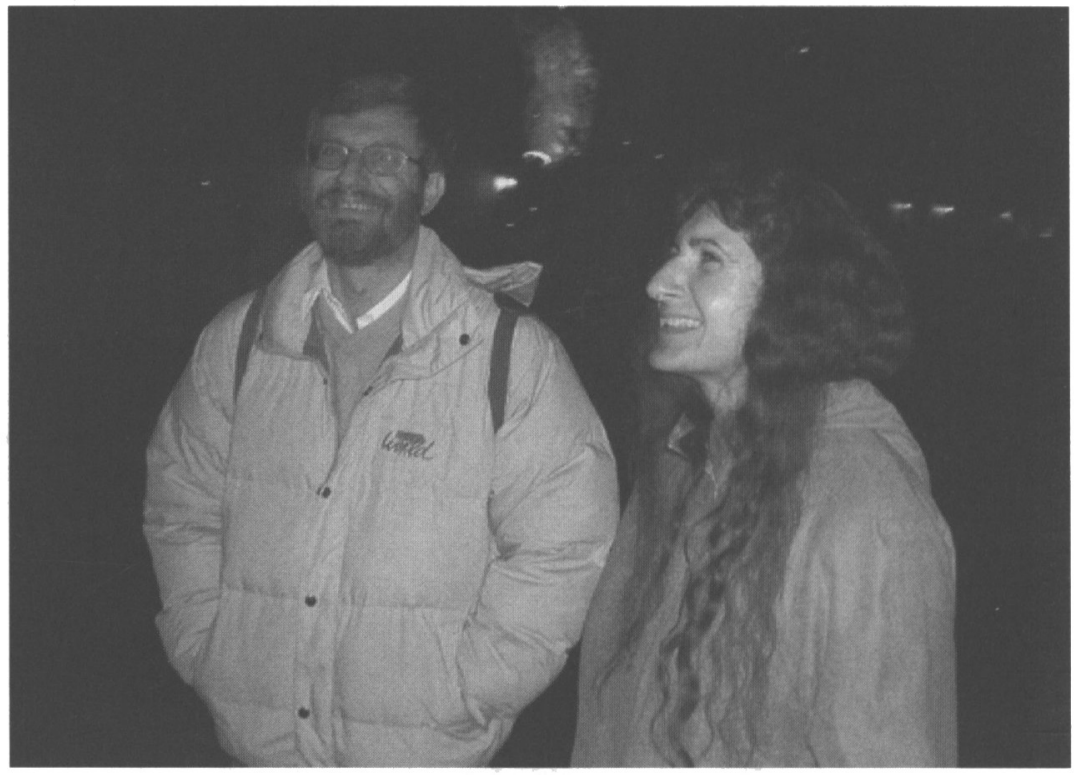

Jan Cuypers and Joyce Guzik 